\title{
Nuclear structure of the transactinides - investigated by decay spectroscopy
}

\author{
Fritz Peter Heßberger ${ }^{1,2, a}$ \\ ${ }^{1}$ GSI Helmholtzzentrum für Schwerionenforschung GmbH, Planckstraße 1, 64291 Darmstadt, \\ Germany \\ ${ }^{2}$ Helmholtz-Institut Mainz, Johann-Joachim-Becherweg 45, 55128 Mainz, Germany
}

\begin{abstract}
Superheavy elements owe their stability due to a subtle balance between the disruptive Coulomb force and the attractive nuclear forces. Thus they represent an ideal laboratory to study basic interactions. The essential tools are detailed investigations of radioactive decay properties and nuclear structure of superheavy nuclei. The results of those studies will deliver valuable input to improve theoretical models. To fulfill this demand conclusive data of high quality are necessary, which is presently not so easy to meet due to small production cross sections and technical limitations (beam intensities, detection probabilities). Possibilities and problems concerning extraction of decay properties and nuclear structure information on the basis of a low number of observed decay events will be discussed for three illustrative examples, ${ }^{257} \mathrm{Rf},{ }^{257} \mathrm{Lr}$, and ${ }^{288} \mathrm{Fl}$.
\end{abstract}

\section{Introduction}

About fifty years ago first attempts to extent the nuclear shell model [1,2] into regions far beyond the heaviest known doubly magic nucleus, ${ }^{208} \mathrm{~Pb}(Z=82, N=126)$, were undertaken. They resulted in the prediction of spherical proton $(Z)$ and neutron $(N)$ shell closures at $Z=114$ and $N=184[3,4]$, which came along with high shell-correction energies in the order of $-8 \mathrm{MeV}$ (see, e.g., Ref. [5]) leading to a high stabilization of nuclei in that region against prompt disruption. Early calculations [6] predicted, e.g. for ${ }^{298} \mathrm{Fl}(Z=114, N=184)$ a partial fission half-life of $2 \cdot 10^{19}$ years. The expected long-lived nuclei around $Z=114$ and $N=184$ were denoted "superheavy". These theoretical findings initiated tremendous efforts from experimental side to produce those "Superheavy Nuclei" (SHN) and to investigate their properties. Up to now synthesis of elements up to $Z=118$ has been reported (see, e.g., Ref. [7]), and discovery of elements $Z>112$ was recently approved by the International Union for Pure and Applied Chemistry (IUPAC) [8-10]. The decay properties of the reported isotopes indicate the existence of a region of shell stabilized nuclei in the transactinide region towards $N=184$, although the "center" has not yet been reached. Information on the strength of the possible shells is still scarce.

From the theoretical side the situation is somewhat more complicated: while macroscopic-microscopic approaches based on the nuclear drop model [11] and the Strutinsky shell-correction method [12] agree in $Z=114$ and $N=184$ as proton and neutron shell closures (see, e.g., Refs. [13, 14]), calculations using self-consistent models like Skyrme-Hartree-Fock-Bogoliubov (SHFB) calculations or relativistic mean-field models

\footnotetext{
a e-mail: f.p.hessberger@gsi.de
}

(C) The Authors, published by EDP Sciences. This is an Open Access article distributed under the terms of the Creative Commons Attribution License 4.0 (http://creativecommons.org/licenses/by/4.0/). 
(RMF) using NL3, NL-Z2 or NL-Z parametrizations [15, 16] disagree in locating the proton and neutron shells. Some predict $Z=120$ as proton shell closure, while others predict $Z=114$ or $Z=126$. Skyrme-force based calculations agree in $N=184$ as neutron shell closure, while the RMF calculations favour $N=172$. Evidently, however, all these parametrizations result in a wide area of large shell effects. It has, however, already been discussed extensively by Bender et al. [17] that $Z$ and $N$ where the shells occur strongly depend on details in the description of the underlying forces, specifically on the values for the effective masses, $m^{*}$, and the strength of the spin-orbit interaction. It has been emphasized in [17] that the energy gap between the spin-orbit partners $2 f_{5 / 2}$ and $2 f_{7 / 2}$ determines whether the proton shell occurs at $Z=114$ or $Z=120$. Under this aspect prediction of different shell closures in different models may be regarded rather as a feature of "fine tuning" of the models than as a principle disagreement. More detailed knowledge of properties and structure of heaviest nuclei is thus undoubtedly decisive for improvements of theoretical models. Therefore such investigations will become in future even more important than synthesis of new elements.

The experimental success in the synthesis of isotopes up to $Z=118$, and the recent and still ongoing theoretical attempts to use effective nucleon-nucleon interactions may be suited to change the point of view of SHN research. While so far the hunt for the spherical proton and neutron shells was in the foreground, it should be stressed out more in future that nucleon shells and enhanced stability are the result of a delicate balance between the Coulomb force and nuclear forces. Since SHN are unstable against prompt disruption using a macroscopic liquid drop picture, their existence and properties are essentially governed by subtleties of the nuclear forces. Thus they represent an ideal laboratory to study the latter. The resulting vision is a common description of phenomena in the "macro-cosmos" (cosmology, neutron stars) and the "micro-cosmos" (nuclei, sub-nuclear phenomena). Such ideas are not new, they were discussed already by, e.g., Chabanat et al. $[18,19]$. Yet, it has to be stated, that presently we are far away from such a description. Nevertheless, it is suggested as a way to go, and detailed investigations of the properties of SHN will be a valuable tool to reach that goal.

To promote scientific progress, a critical debate about theoretical models is necessary. Experimental results have not just to be discussed with respect to confirm theoretical predictions, as it is often done. Rather, they have to be regarded as firm tests of theoretical predictions, and discrepancies (e.g. in $Q_{\alpha}$-values, excitation energies and ordering of nuclear levels, fission half-lives, etc.) clearly point to deficiencies that have to be overcome. Nevertheless, the quality of theoretical predictions is often high enough for a classification of experimental data, e.g. tentative isotope assignments on the basis of decay $Q$-values, tentative nuclear level and spin assignments on the basis of predicted energies, and ordering of nuclear levels. The reason is that in general a sufficiently large amount of data of sufficient quality is available to achieve an impression of the predictive power of a model. Regarding this feature, in a first step the experimental assignments seem reasonable when in line with theoretical predictions within their limits of validity, while those leading to too extreme deviations seem rather improbable.

The discussions above lead to the trivial statement that developments of experiments and theories have to go hand-in-hand and to influence each other, but always having a critical view on the results.

From the experimental side refined techniques to deliver data of high quality are required, as it does not make any sense to draw any conclusions, also concerning the validity of the theoretical predictions, from data of low quality, which are not conclusive by themselves. Unfortunately, often only "low quality" data are available. In such cases, however, it would not be wise to discard the results, but they have to be presented critically and deficiencies 

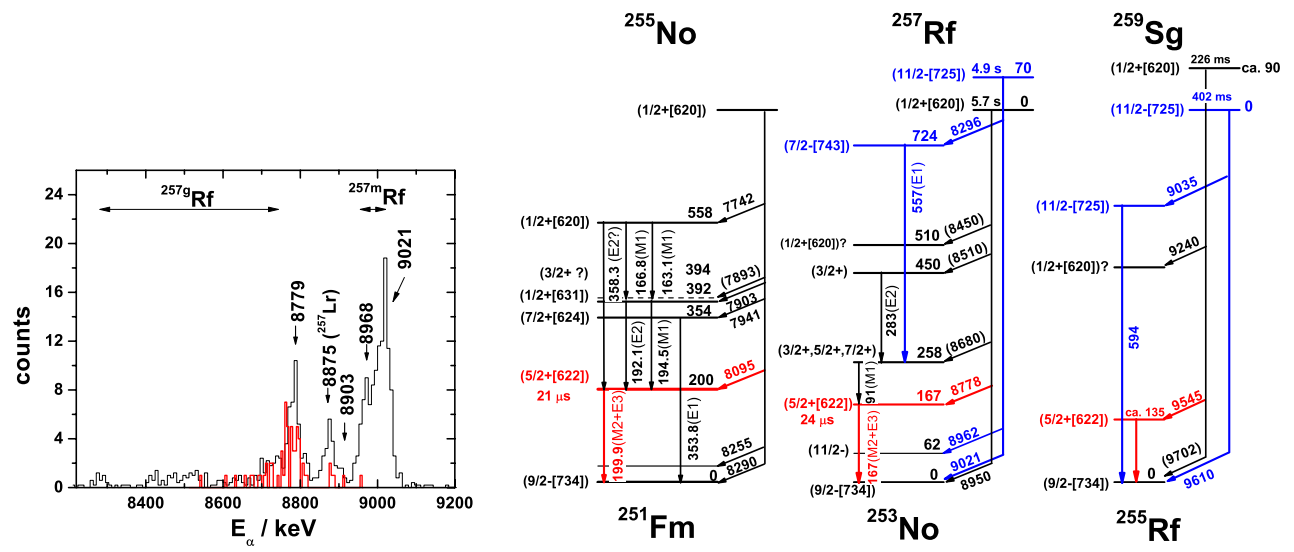

Figure 1. Left side: alpha spectra of ${ }^{257} \mathrm{Rf}$; black line: direct production via ${ }^{208} \mathrm{~Pb}\left({ }^{50} \mathrm{Ti}, n\right){ }^{257} \mathrm{Rf}$; red line: indirect production via ${ }^{208} \mathrm{~Pb}\left({ }^{58} \mathrm{Fe}, n\right){ }^{265} \mathrm{Hs} \stackrel{\alpha}{\rightarrow}{ }^{261} \mathrm{Sg}_{\rightarrow}^{\alpha}{ }^{257} \mathrm{Rf}$.

Figure 2. Right side: decay schemes of ${ }^{255} \mathrm{No},{ }^{257} \mathrm{Rf}$, and ${ }^{259} \mathrm{Sg}$.

and problems connected with their interpretation must not be concealed but have to be clearly pointed out. Only under this aspect they represent valuable information and challenge for improvements to obtain data of better quality. Insofar experimental work is a process of learning and gradual improvement of knowledge step by step. Sometimes numerous experiments spanning a couple of decades were performed, and despite large progress a final conclusion on decay properties and nuclear structure still has not yet been reached. An example are decay studies of ${ }^{257} \mathrm{Rf}$ which will be discussed in the following.

\section{Alpha and EC decay studies of ${ }^{257} \mathrm{Rf}$}

The isotope ${ }^{257} \mathrm{Rf}$ was first synthesized by Ghiorso et al. [20] in the reaction ${ }^{249} \mathrm{Cf}\left({ }^{12} \mathrm{C}, 4 n\right){ }^{257} \mathrm{Rf}$ using the He-jet technique. They observed a complex $\alpha$ spectrum with energies in the range (8.5-9.0) $\mathrm{MeV}$ and reported a half-life of (4.5 \pm 1.0$)$ s. Some years later, Bemis et al. [21] confirmed the results. In addition, they also measured photons in coincidence with $\alpha$ particles and observed $K X$ rays of energies agreeing with those expected for nobelium. It thus was the first direct $Z$-identification of a transactinide element. Part of the $K X$ rays were observed in delayed coincidence with $\alpha$ particles; this was interpreted as due to populating an isomeric state in ${ }^{253}$ No by the $\alpha$ decay. It was settled at an excitation energy of $\approx 300 \mathrm{keV}$ and its half-life was given as $T_{1 / 2}=(31.1 \pm 4.1) \mu$ s [21]. At SHIP this isotope was produced in the reaction ${ }^{208} \mathrm{~Pb}\left({ }^{50} \mathrm{Ti}, n\right){ }^{257} \mathrm{Rf}[22,23]$ (direct production) and by $\alpha$ decay of ${ }^{265} \mathrm{Hs}\left(\stackrel{\alpha}{\rightarrow}{ }^{261} \mathrm{Sg} \stackrel{\alpha}{\rightarrow}{ }^{257} \mathrm{Rf}\right.$ ) $[23,24]$ (indirect production). Comparison of the $\alpha$ spectra from the direct and indirect production revealed that the $\alpha$ lines of highest energy $(9021,8968 \mathrm{keV})$ were missing in the indirect production (see Fig. 1). It was concluded that these $\alpha$ lines stem from the decay of an isomeric level, not populated by the $\alpha$ decay of ${ }^{261} \mathrm{Sg}$. In addition $\alpha$ transitions at $E_{\alpha}<8.5 \mathrm{MeV}$ could be identified, which were not accessible in the previous studies due to $\alpha$ background from lighter isotopes [20, 21]. In [23] also first decay schemes of $257 \mathrm{~g}, 257 \mathrm{~m}$ Rf were presented. Due to interpretation of a weak $\alpha$ line at $8903 \mathrm{keV}$ (see Fig. 1) as the ground-state to ground-state (gs-gs) transition, the $5 / 2^{+}[622]$ Nilsson level was located at $E^{*}=122 \mathrm{keV}$, leading to a discrepancy overseen in [23]: the $5 / 2^{+}$[622] level was identified as an isomeric level in the lighter $N=151$ isotones $\left({ }^{249} \mathrm{Cf},{ }^{251} \mathrm{Fm}\right)$ decaying to the ground state 
by highly converted $M 2$ transitions (with $E 3$ admixtures). Thus it could be also assumed in ${ }^{253} \mathrm{No}$ as the source of the delayed $K X$ rays observed in [21]; its excitation energy of $122 \mathrm{keV}$, assigned in [23], however, is below the $K$ binding energy in nobelium. Subsequently, more precise measurements clarified this problem and finally settled the excitation energy of the $5 / 2^{+}[622]$ state at $167 \mathrm{keV}$ by measuring $\gamma$ transitions $5 / 2^{+}[622] \rightarrow 9 / 2^{-}[734](\mathrm{gs})$ in delayed coincidence with $\alpha$ particles [25].

The long-lived isomeric state had been attributed as $11 / 2^{-}$[725] in [23] on the basis of systematics of Nilsson levels in $N=153$ isotones and calculated levels schemes [26], as it was the only predicted level that allowed for highly hindered internal transition into the ground state necessary to form an isomer decaying by $\alpha$ emission with a half-life of about $4 \mathrm{~s}$.

Recent studies at SHIP [27] resulted in the observation of two $\alpha-\gamma$-coincidences $(8296 \mathrm{keV}, 557 \mathrm{keV})$, which were attributed to the $\alpha$ decay of ${ }^{257 m} \mathrm{Rf}$, populating the $7 / 2^{-}$[743] Nilsson level in ${ }^{253} \mathrm{No}$ and then decaying into the isomeric $5 / 2^{+}$[622] level [27] as shown in Fig. 2.

Another feature of the recent study [27] was investigation of electron capture (EC) decay of ${ }^{257} \mathrm{Rf}$ by measuring delayed coincidences between conversion electrons (CE) from decay of excited levels in ${ }^{257} \mathrm{Lr}$ populated by the EC decay and $\alpha$ decays of ${ }^{257} \mathrm{Lr}$. Comparison of the decay data of ${ }^{257} \mathrm{Lr}$ resulting from the direct and indirect [25] production of ${ }^{257} \mathrm{Rf}$ as well as from production of ${ }^{257} \mathrm{Lr}$ by $\alpha$ decay of ${ }^{261} \mathrm{Db}[25,28]$ revealed significant differences in the energy and time distributions of the $\alpha$ decays of ${ }^{257} \operatorname{Lr}$ (see Fig. 3), which were interpreted as $\alpha$ decays from two low-lying levels in ${ }^{257} \mathrm{Lr}$. The lower energy $(8811 \mathrm{keV})$ was attributed to the decay of the isomeric state with $T_{1 / 2}=\left(0.20_{-0.06}^{+0.16}\right) \mathrm{s}$ and tentatively assigned as $1 / 2^{-}$[521], the higher one $(8878 \mathrm{keV})$ to the decay of the ground state having $T_{1 / 2}=\left(1.24_{-0.36}^{+0.85}\right) \mathrm{s}$ (obtained from the ${ }^{261} \mathrm{Db}^{257} \mathrm{Lr}$-correlations) and tentatively assigned as $7 / 2^{-}[514]$ or $9 / 2^{+}[624]$ in Ref. [27] as shown in Fig. 4a.

The occurrence of two low-lying states decaying by $\alpha$ emission is not unexpected, as such states have been reported previously in the lighter odd-mass isotopes ${ }^{253} \mathrm{Lr}$ [30] and ${ }^{255} \operatorname{Lr}[31,32]$. The situation is shown in Fig. 5 where the experimental results are compared with theoretical predictions [29]. Of specific interest are here the relative positions of the $1 / 2^{-}$[521] and the $7 / 2^{-}$[514] states. The former originates from the $2 f_{5 / 2}$ subshell, the latter from the $2 f_{7 / 2}$. As pointed out in [17] (see above) the gap between the spinorbit partners $2 f_{5 / 2}-2 f_{7 / 2}$ defines the location of the spherical proton shells with $Z=114$ or $Z=120$. Therefore identification and determination of their excitation energies in the lawrencium isotopes already delivers valuable data for determining the spin-orbit coupling and thus for prediction of the location of the spherical proton shell. Clearly more data are required.

Another result of the analysis of the delayed CE- $\alpha$ coincidences was the observation of some photons in prompt coincidence with CE. Evidently, at a low background rate, three photons fit to energies of lawrencium $K_{\alpha 1}$ and $K_{\beta 1} X$ rays [see Fig. 4b], supporting the EC decay of ${ }^{257} \mathrm{Rf}$ and also showing the possibility to use $K X$ rays from EC decay for $Z$-identification of SHN, as recently demonstrated for EC decay of ${ }^{258} \mathrm{Db}$ [33].

\section{Alpha-decay properties of ${ }^{288} \mathrm{FI}$}

Nuclear structure investigations require clean measurements of nuclear transitions. In case of measuring $\alpha$ particles this feature might be difficult to fulfil sometimes, when the technique of implanting nuclei into Si-detectors, commonly used in SHN research, is applied. If $\alpha$ decay populates excited daughter levels that decay by internal conversion energy summing between $\alpha$ particles and CE will occur [34]. The result will be a rather broad distribution of $\alpha$-particle 

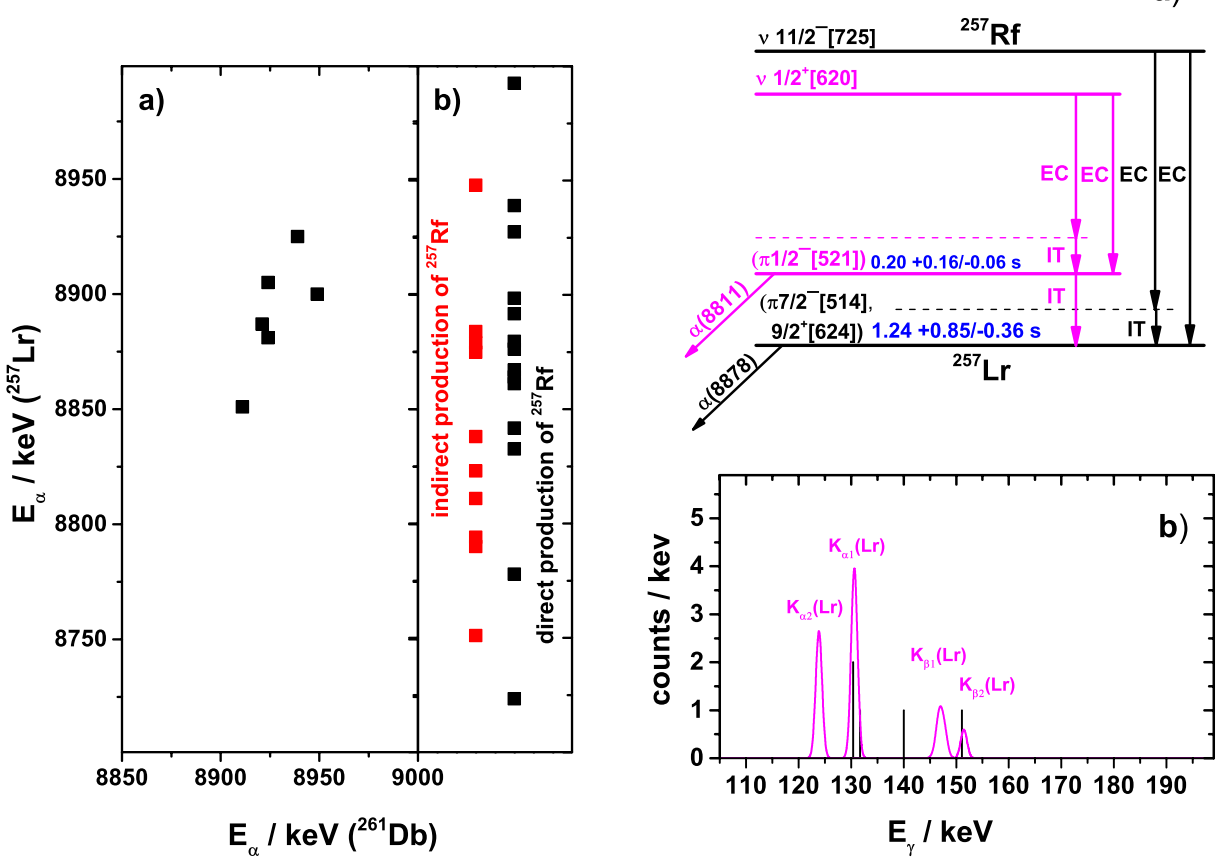

Figure 3. Left side: (a) two-dimensional plot of $\alpha\left({ }^{261} \mathrm{Db}\right)-\alpha\left({ }^{257} \mathrm{Lr}\right)$ correlations; (b) energy distributions of $\alpha$ decays of ${ }^{257} \mathrm{Lr}$ following EC decay of ${ }^{257} \mathrm{Rf}$, either from direct production or indirect production.

Figure 4. Right side: (a) suggested EC decay schemes of ${ }^{257 g, 257 \mathrm{~m}} \mathrm{Rf}$; (b) spectrum of photons measured in prompt coincidence with CE preceding $\alpha$ decays of ${ }^{257} \operatorname{Lr}$ within $\Delta t \leq 1.5 \mathrm{~s}$.
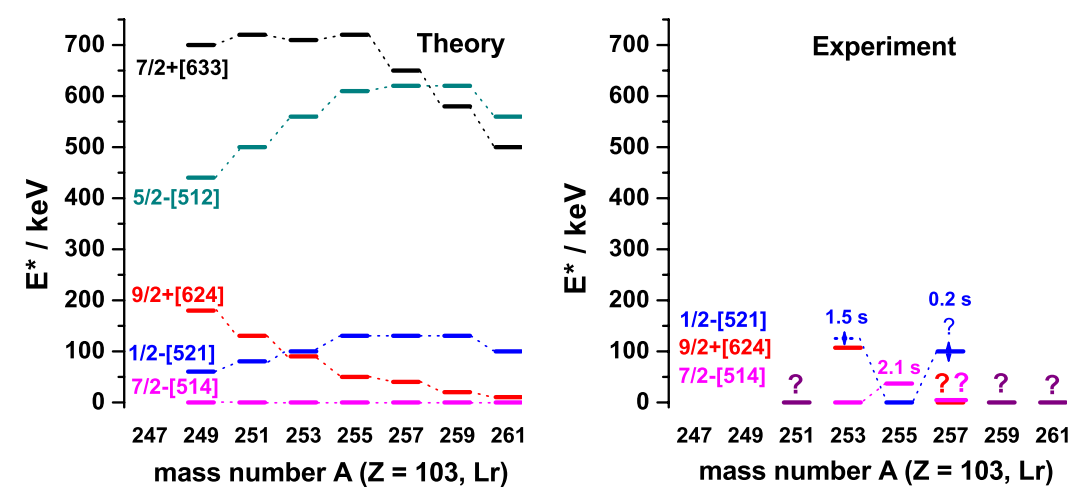

Figure 5. Predicted [29] and (tentative) experimental level schemes of odd-mass Lr isotopes.

energies, washing out peak structures, as shown in [35] for the case of ${ }^{255}$ No. At low number of observed events in the extreme case just a broad distribution of $\alpha$ events might be observed. As a consequence one may obtain a superposition of $\alpha$ energy distributions of neighbouring isotopes or of $\alpha$ decays from different isomeric states in a specific nucleus.

In the range of $Z>112$ it was recently shown [36, 37] that a postulated link [38] between the $\alpha$-decay chain starting from ${ }^{293} 117$ (produced via ${ }^{249} \mathrm{Bk}\left({ }^{48} \mathrm{Ca}, 4 n\right){ }^{293} 117$ ) and from ${ }^{289} 115$ 

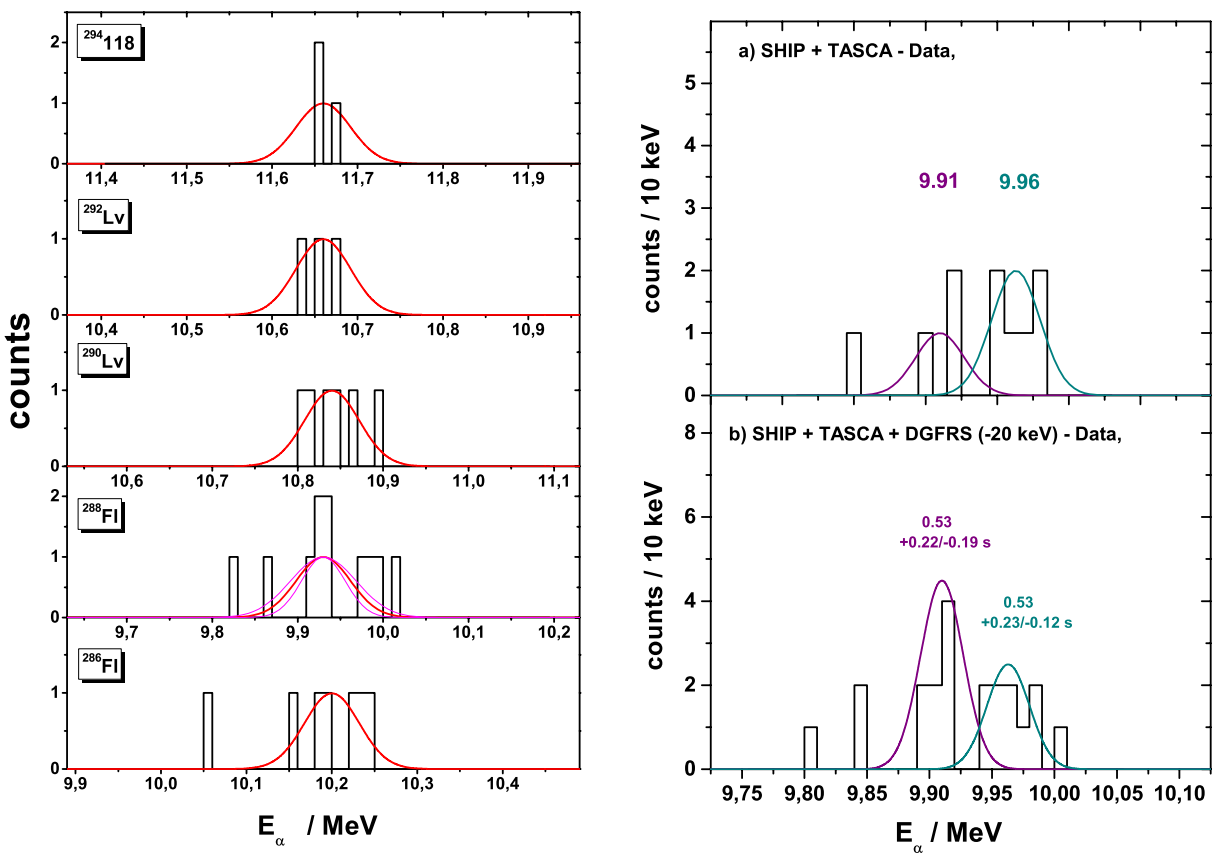

Figure 6. Left side: alpha spectra of even-even isotopes observed at the DGRFS (see text for details).

Figure 7. Right side: alpha spectrum of ${ }^{288} \mathrm{Fl}$; (a) sum of SHIP and TASCA data; (b) sum of SHIP, TASCA, and DGFRS data (see text for details.)

(produced via $\left.{ }^{243} \mathrm{Am}\left({ }^{48} \mathrm{Ca}, 2 n\right)^{289} 115\right)$ does not exist. This link was built on assigning all "short" decay chains observed in the latter reaction with levity to the decay of the same state in ${ }^{289} 115$ (see [39] for details).

Another example for inconsistencies in the $\alpha$ spectra of isotopes in the region $Z>112$ is the even-even nucleus ${ }^{288} \mathrm{Fl}$. Alpha-spectra of even-even nuclei usually have a quite simple structure as the $0^{+} \rightarrow 0^{+}$transition between the ground-states dominates due to the highest $Q$-values and the absence of spin hindrance. In Fig. 6 the $\alpha$ spectra of all $Z>112$ eveneven nuclei observed in experiments at the Dubna gas-filled separator (DGFRS) are shown. To avoid distortions due to worse energy resolution of $\alpha$ particles registered as sum events in the focal plane (stop) detector and the box detector, only events with full energy release in the stop detector are considered. The red lines represent Gaussians calculated using a detector resolution of $75 \mathrm{keV}$ (FWHM), the mean value given for the range (60-90) keV for the stop detector in [43]. The distributions for ${ }^{294} 118$ [40, 41], ${ }^{292,290} \mathrm{Lv}$ [40-42], and also ${ }^{286} \mathrm{Fl}[40,44]$ are justifiable with a single $\alpha$ line, while for ${ }^{288} \mathrm{Fl}[42,43]$ this is questionable. To emphasize this feature also the Gaussians using detector resolutions of $60 \mathrm{keV}$ and $90 \mathrm{keV}$ are shown by the magenta lines. Indeed, a possible fine structure in the $\alpha$ decay of ${ }^{288} \mathrm{Fl}$ has also very recently mentioned by Oganessian and Utyonkov [7], however, without drawing definite conclusions. ${ }^{288} \mathrm{Fl}$ was also observed in experiments at the gas-filled separator TASCA [45] and the velocity filter SHIP [46] at GSI. The results are shown in Fig. 7a. The detector resolutions in these experiments were significantly better, $25 \mathrm{keV}$ (FWHM) [45] and $24 \mathrm{keV}$ (FWHM) [46]. The data also indicate the existence of two $\alpha$ lines. The existence of two $\alpha$ lines becomes even more evident when the results of all experiments are summed, as shown in Fig. 7b. (The results from DGFRS have been scaled by $-20 \mathrm{keV}$ to match 
apparent differences in the calibrations.) Mean energies and half-lives of $(9.11 \pm 0.01) \mathrm{MeV}$, $\left(0.53_{-0.19}^{+0.22}\right) \mathrm{s}$ and $(9.96 \pm 0.01) \mathrm{MeV},\left(0.53_{-0.12}^{+0.23}\right) \mathrm{s}$ are obtained.

The $\alpha$ spectrum of ${ }^{288} \mathrm{Fl}$ presently cannot be explained satisfactorily: although the energy difference between the two lines is similar to typical differences for the $0^{+}$ground-state and the first $2^{+}$state in highly deformed heavy nuclei [47], this feature cannot explain the intensity ratio of the two lines. The intensities for decay into the $2^{+}$level are typically only $10-20 \%$ of those of decays into the $0^{+}$ground state, and the $2^{+} \rightarrow 0^{+} E 2$-transitions are highly converted. Energy summing between $\alpha$ particles and CE will shift the energies towards the energy of transition into the ground state and thus the lines would not be resolved. Another possibility could be the existence of a very low lying $1^{-}$vibrational state in the daughter nucleus ${ }^{284} \mathrm{Cn}$, as existing, e.g., in ${ }^{222} \mathrm{Ra}\left(E^{*}=242.13 \mathrm{keV}\right)$ and ${ }^{224} \mathrm{Ra}$ $\left(E^{*}=215.99 \mathrm{keV}\right)$ [47]; those decay by weakly converted $E 1$ transitions into the ground state [47]; however, $\alpha$-decay branchings into these levels are very low $(<0.015)$.

As ${ }^{288} \mathrm{Fl}$ is one member of a "mesh of isotopes" a simple assignment to neighbouring odd-mass nuclei seems not possible as it is followed by a short-lived fission activity. As the daughter products of the neighbouring isotopes $\left({ }^{289} \mathrm{Fl},{ }^{287} \mathrm{Fl}\right)$ are long-lived $\alpha$ emitters [7] this would imply a shorter fission half-life of an odd-mass nucleus compared to a neighbouring even-even nucleus, which contradicts our knowledge of fission properties of SHN. A third possibility would be existence of an isomeric state, but with respect to the existing data (including the same half-lives within the error bars) such an assumption is not well based. To summarize: one has to state a strange behavior of the $\alpha$ spectrum of ${ }^{288} \mathrm{Fl}$, but no simple explanation for it can be given so far. Careful studies are required to draw final conclusions, and also to exclude that this behavior is not just an artifact, caused by a "random" scattering of the data and a rather low $(\approx 20$ events) number of observed $\alpha$ decays.

\section{Conclusion}

It has been shown for the case of ${ }^{257} \mathrm{Rf}^{257} \mathrm{Lr}$ that valuable nuclear structure information can be extracted by careful and critical analysis of the data and considering the complete physical enviroment even on the basis of a quite low number of observed events. Certainly they do not deliver a complete picture, but mark the road for further investigations. The case of ${ }^{288} \mathrm{Fl}$, on the other hand, shows that a blinkered data analysis (see e.g. [48]) can lead to missing essential features. Indeed, the situation is sometimes ambivalent, as often "unexpected" results later turned out to be artifacts (see, e.g., discussion on ${ }^{255} \mathrm{Rf}$ in $[23,30]$ ). These facts, however, simply corroborate the demand for high-quality data free of ambiguities before drawing conclusions on identification, decay properties, or nuclear structure of superheavy nuclei.

\section{References}

[1] M. Göppert-Mayer, Phys. Rev. 74, 235 (1948).

[2] O. Haxel et al., Phys. Rev. 75, 1769 (1949).

[3] A. Sobiczewski et al., Phys. Lett. 22, 500 (1966).

[4] H. Meldner, Arkiv för fysik 36, 593 (1967).

[5] S.G. Nilsson et al., Phys. Lett. 28B, 458 (1969).

[6] S.G. Nilsson et al., Nucl. Phys. A115, 545 (1968).

[7] Yu.Ts. Oganessian and V.K. Utyonkov, Nucl. Phys. A944, 62 (2015).

[8] R.C. Barber et al., Pure Appl. Chem. 83, 1485 (2011).

[9] P.J. Karol et al., Pure Appl. Chem. 88, 139 (2016). 
[10] P.J. Karol et al., Pure Appl. Chem. 88, 155 (2016).

[11] C.F. von Weizsäcker, Z. Phys. 96, 461 (1935).

[12] V.M. Strutinsky, Nucl. Phys. A95, 442 (1967).

[13] R. Smolanczuk and A. Sobiczewski, Proc. EPS Conf. Low Energy Nuclear Dynamics, St. Petersburg 1995, eds. Yu.Ts. Oganessian et al. (World Scientific, Singapore, New Jersey, London, Hong Kong, 1995), p. 313.

[14] P. Möller et al., At. Data and Nucl. Data Tab. 59, 185 (1995).

[15] K. Rutz et al., Proc. of the Second Int. Conf. Fission and Properties of NeutronRich Nuclei, St. Andrews 1999, eds. J.H. Hamilton et al. (World Scientific, Singapore, New Jersey, London, Hong Kong, 2000), p. 449.

[16] M. Bender et al., Rev. Mod. Phys. 75, 121 (2003).

[17] M. Bender et al., Phys. Rev. C 60, 034304 (1999).

[18] E. Chabanat et al., Nucl. Phys. A627, 710 (1997).

[19] E. Chabanat et al., Nucl. Phys. A635, 231 (1998).

[20] A. Ghiorso et al., Phys. Rev. Lett. 22, 1317 (1969).

[21] C.E. Bemis et al., Phys. Rev. Lett 31, 647 (1973).

[22] F.P. Heßberger et al., Z. Phys. A322, 557 (1985).

[23] F.P. Heßberger et al., Z. Phys. A359, 415 (1997).

[24] S. Hofmann et al., Z. Phys. A350, 277 (1995).

[25] B. Streicher et al., Eur. Phys. J. A45, 275 (2010).

[26] S. Cwiok et al., Nucl. Phys. A 573, 356 (1997).

[27] F.P. Heßberger et al., Eur. Phys. J. A52, 192 (2016).

[28] Z. Gan et al., Eur. Phys. J. A20, 385 (2004).

[29] A. Parkhomenko and A. Sobiczewski, Acta Phys. Pol. B 35, 2447 (2004).

[30] F.P. Heßberger et al., Eur. Phys. J. A12, 57 (2001).

[31] A. Chatillon et al., Eur. Phys. J. A30, 397 (2006).

[32] S. Antalic et al., Eur. Phys. J. A38, 219 (2008).

[33] F.P. Heßberger et al., GSI Scientific Report 2014, MU-NUSTAR-SHE-P-09, 177 (2015).

[34] F.P. Heßberger et al., Nucl. Instr. Meth. A274, 522 (1989).

[35] M. Asai, F.P. Heßberger, A. Lopez-Martens, Nucl. Phys. A944, 308 (2015).

[36] U. Forsberg et al., Nucl. Phys. A953, 117 (2016).

[37] U. Forsberg et al., Phys. Lett. B760, 293 (2016).

[38] Yu.Ts. Oganessian et al., Phys. Rev. C 87, 054621 (2013).

[39] U. Forsberg, PhD Thesis, Lund University, ISBN 978-91-7623-813-4 (2016).

[40] Yu.Ts. Oganessian et al., Phys. Rev. C 74, 044602 (2006).

[41] Yu.Ts. Oganessian et al., Phys. Rev. Lett. 109, 162501 (2012).

[42] Yu.Ts. Oganessian et al., Preprint E7-2004-160 (2004).

[43] Yu.Ts. Oganessian et al., Phys. Rev. C 69, 054607 (2004).

[44] Yu.Ts. Oganessian et al., Phys. Rev. C 70, 064609 (2004).

[45] J.M. Gates et al., Phys. Rev. C 83, 054018 (2011).

[46] S. Hofmann et al., Eur. Phys. J. A48, 62 (2012).

[47] R.B. Firestone et al., Table of Isotopes, 8th Edition (Wiley \& Sons, New York, 1996).

[48] S. Hofmann et al., Eur. Phys. J. A52, 180 (2016). 